\title{
Self-Regulation: A Critical Construct in Research and Application with Children and Families
}

\author{
Ronald J. Prinz ${ }^{1}$
}

Published online: 19 February 2019

(c) Springer Science+Business Media, LLC, part of Springer Nature 2019

This special journal issue in Clinical Child and Family Psychology Review focuses on self-regulation as it relates to research and application with children and families. The Guest Co-Editors for this special journal issue are Dr. Stephanie Jones, from the Graduate School of Education and the Center for the Developing Child at Harvard University, and Dr. Matthew Sanders, who directs the Parenting and Family Support Centre at the University of Queensland. The impetus for this special issue stems from the observation that self-regulation is a dominant concept in areas of inquiry related, for example, to child development, parenting, family/school-based interventions, and issues in the implementation of evidence-based programs (EBPs).

Self-regulation, a complex and multidimensional construct, can be applied to many problems and contexts. Accordingly, the scientific literature addressing various facets and applications of self-regulation is too large to be covered in a single journal issue or even a book volume. The goals of this special issue were to sample some of the more prominent work on self-regulation pertaining to research and intervention applied to children, as well as parenting and families, discuss conceptual and methodological progress, and offer recommendations for fruitful directions.

Research on child self-regulation commonly addresses three facets-cognitive, social/behavioral, and emotional self-regulation. Related concepts and skills include planning, problem-solving, empathy and perspective-taking, interactional adeptness, behavioral inhibition, self-calming, and anger control. In the articles focusing primarily on self-regulation in children, the contributing authors review research and illuminate issues about (1) executive function and effortful control as foundational constructs; (2) the roles of self-regulation in language development during early childhood; (3) digital technology in the assessment of selfregulation in classroom contexts; and (4) development of healthy habits in childhood.

Parents undoubtedly play a major role in how young children, as well as older children, acquire and refine selfregulatory skills and capacities. The contributors elaborate on the specific ways in which parents facilitate or inhibit the development of self-regulation, on how interventions can strengthen parenting to promote child self-regulation, and on invoking self-regulation in parents to further empower and support parenting growth.

Finally, self-regulation is examined in broader ways applied to larger systems, for example, in the training of practitioners who deliver EBPs to families, in a model for successful and sustainable implementation of programming, and in the measurement and implementation of self-regulation intervention components in the context of low- and middle-income countries.

The many authors who contributed to the 10 articles in this special issue generated a wealth of information and ideas in the hope that readers will derive benefits that progress our knowledge and application of self-regulation for the well-being of children and families.

Publisher's Note Springer Nature remains neutral with regard to jurisdictional claims in published maps and institutional affiliations.
Ronald J. Prinz

prinz@mailbox.sc.edu

1 Department of Psychology, University of South Carolina, Columbia, SC 29208, USA 